\title{
Examining the effect of Withania somnifera supplementation on muscle strength and recovery: a randomized controlled trial
}

\author{
Sachin Wankhede ${ }^{1}$, Deepak Langade ${ }^{2}$, Kedar Joshi $^{3}$, Shymal R. Sinha ${ }^{4}$ and Sauvik Bhattacharyya ${ }^{5^{*}}$
}

\begin{abstract}
Background: Withania somnifera (ashwagandha) is a prominent herb in Ayurveda. This study was conducted to examine the possible effects of ashwagandha root extract consumption on muscle mass and strength in healthy young men engaged in resistance training.

Methods: In this 8-week, randomized, prospective, double-blind, placebo-controlled clinical study, 57 young male subjects (18-50 years old) with little experience in resistance training were randomized into treatment (29 subjects) and placebo (28 subjects) groups. Subjects in the treatment group consumed $300 \mathrm{mg}$ of ashwagandha root extract twice daily, while the control group consumed starch placebos. Following baseline measurements, both groups of subjects underwent resistance training for 8 weeks and measurements were repeated at the end of week 8 . The primary efficacy measure was muscle strength. The secondary efficacy measures were muscle size, body composition, serum testosterone levels and muscle recovery. Muscle strength was evaluated using the 1-RM load for the bench press and leg extension exercises. Muscle recovery was evaluated by using serum creatine kinase level as a marker of muscle injury from the effects of exercise.
\end{abstract}

Results: Compared to the placebo subjects, the group treated with ashwagandha had significantly greater increases in muscle strength on the bench-press exercise (Placebo: $26.4 \mathrm{~kg}, 95 \% \mathrm{Cl}, 19.5,33.3$ vs. Ashwagandha: $46.0 \mathrm{~kg}, 95 \% \mathrm{Cl} 36.6,55.5 ; p=0.001$ ) and the leg-extension exercise (Placebo: $9.8 \mathrm{~kg}, 95$ \% Cl, 7.2,12.3 vs. Ashwagandha: $14.5 \mathrm{~kg}, 95 \% \mathrm{Cl}, 10.8,18.2 ; p=0.04$ ), and significantly greater muscle size increase at the arms (Placebo: $5.3 \mathrm{~cm}^{2}, 95 \% \mathrm{Cl}, 3.3,7.2$ vs. Ashwagandha: $8.6 \mathrm{~cm}^{2}, 95 \% \mathrm{Cl}, 6.9,10.8 ; p=0.01$ ) and chest (Placebo: $1.4 \mathrm{~cm}$, $95 \% \mathrm{Cl}, 0.8,2.0$ vs. Ashwagandha: $3.3 \mathrm{~cm}, 95 \% \mathrm{Cl}, 2.6,4.1 ; p<0.001)$. Compared to the placebo subjects, the subjects receiving ashwagandha also had significantly greater reduction of exercise-induced muscle damage as indicated by the stabilization of serum creatine kinase (Placebo: $1307.5 \mathrm{U} / \mathrm{L}, 95 \% \mathrm{Cl}, 1202.8,1412.1$, vs. Ashwagandha: $1462.6 \mathrm{U} / \mathrm{L}, 95 \% \mathrm{Cl}, 1366.2,1559.1 ; p=0.03)$, significantly greater increase in testosterone level (Placebo: $18.0 \mathrm{ng} / \mathrm{dL}, 95 \% \mathrm{Cl},-15.8,51.8$ vs. Ashwagandha: $96.2 \mathrm{ng} / \mathrm{dL}, 95 \% \mathrm{Cl}, 54.7,137.5 ; p=0.004$ ), and a significantly greater decrease in body fat percentage (Placebo: $1.5 \%, 95 \% \mathrm{Cl}, 0.4 \%, 2.6 \%$ vs. Ashwagandha: $3.5 \%$, $95 \% \mathrm{Cl}, 2.0 \%, 4.9 \% ; p=0.03)$.

Conclusion: This study reports that ashwagandha supplementation is associated with significant increases in muscle mass and strength and suggests that ashwagandha supplementation may be useful in conjunction with a resistance training program.

Keywords: Ashwagandha, Adaptogen herbs, Muscle, Muscle strength, Muscle mass, Testosterone, Body fat, Creatine kinase

\footnotetext{
* Correspondence: dr.sauvik.bhattacharyya@gmail.com

${ }^{5}$ Department of Pharmaceutical Technology, NSHM Knowledge Campus, 124 B.L. Saha Road, Kolkata 700053, India

Full list of author information is available at the end of the article
}

\section{Biomed Central}

(c) 2015 Wankhede et al. Open Access This article is distributed under the terms of the Creative Commons Attribution 4.0 International License (http://creativecommons.org/licenses/by/4.0/), which permits unrestricted use, distribution, and reproduction in any medium, provided you give appropriate credit to the original author(s) and the source, provide a link to the Creative Commons license, and indicate if changes were made. The Creative Commons Public Domain Dedication waiver (http://creativecommons.org/publicdomain/zero/1.0/) applies to the data made available in this article, unless otherwise stated. 


\section{Background}

Both the modern medical literature and traditional Ayurveda writings report many potential health benefits of the Ashwagandha herb (Withania somnifera, also known as Indian Ginseng or Winter Cherry) under the rubrics of anti-stress effects, neuroprotective effects, immunomodulatory effects, and rejuvenating effects, via the herb's interplay with the nervous system, the endocrine system, the cardiopulmonary system, the energy production system and the immune system including analgesic, antimicrobial, anti-inflammatory, anti-tumor, anti-stress, anti-diabetic, neuroprotective, immunoprotective and cardioprotective effects [1-7]. This paper focuses on ashwagandha as an ergogenic aid and is the first to present the results of a randomized, doubleblind, placebo-controlled clinical study on ashwagandha's effects, as an adjuvant to a resistance training program, multifariously on muscle strength, muscle hypertrophy, muscle recovery and body composition. We add to the broader literature on ashwagandha's effect on physical performance. This literature has only a small set of published papers [8-10], which is surprising because traditional Ayurveda explicitly advocates the use of ashwagandha toward "bala", which means "strength" in the Sanskrit language [11] .

Ashwagandha is a member of the family of herbs referred to as "adaptogens". The term "adaptogen" is applied to a herb with phytonutrients that regulate metabolism when a body is perturbed by physical or mental stress, and help the body adapt by (a) normalizing system functions, (b) developing resistance to future such stress, and (c) elevating the body's functioning to a higher level of performance [12]. The adaptogen family of herbs has many members, noteworthy among them being ashwagandha, rhodiola, ginseng, schisandra and maca [12]. Adaptogens are used commonly for stress relief, brain health, adrenal health and for ameliorating HPA-axis dysfunction. More recently, adaptogens have started to be used in sports supplements that aim to enhance physical fitness. Recent research has found adaptogens to be promising in this application domain [13-15]. However, the results in this literature are mixed and therefore more research is needed so that we have a better understanding of adaptogens as ergogenic aids [14]. This present study attempts to make a small step towards such an understanding.

A resistance training program consists of exercises that cause skeletal muscles to contract against external resistance. The body often responds to such programs with increased strength and correlated adaptations [16-19]. The present research work was motivated by the hypothesis that ashwagandha supplementation can increase some of these adaptations and gains, thereby serving as a useful adjuvant to a resistance training program.
There are several rationales underlying this hypothesis: Studies in healthy normal adults demonstrated that ashwagandha improves muscular strength/coordination, and cardiorespiratory endurance [8-10]. Ashwagandha's roots are classified as a "rasayana" (rejuvenator), and have been used toward promoting health and longevity, slowing the aging process, revitalizing the body and generally creating a sense of well-being $[4,20]$. Ashwagandha has a wide range of pharmacological effects: it has anxiolytic, hypotensive, sedative, central nervous system, immunomodulatory, analgesic, anti-inflammatory, anti-tumor, anabolic, cardiopulmonary and antioxidant effects [4, 9, 21-27]. It also stimulates respiratory function, causing smooth muscle relaxation, and stimulates thyroid activity [3]. Studies in humans show that ashwagandha is well tolerated and is associated with decreases in cortisol [28], and increases in testosterone [29]. Research suggests that ashwagandha may reduce increases of blood urea nitrogen, lactic acid, corticosterone in response to stress [4], and also reduce the tendency of dopamine receptors in the brain to activate under stress $[3,5,21]$. Ashwagandha contains several active components, which may account for the various mechanisms of action by which it exerts its effects. These include steroidal lactones (withanolides, withaferins), saponins and alkaloids like isopelletierine and anaferine [5, 30].

The adaptogenic properties of ashwagandha raise the possibility of it being an effective ergogenic aid because the strain from exercise can be viewed as a form of stress, with enhanced human physical performance as the corresponding stress response upon ashwagandha supplementation. This study seeks to examine the hypothesis that ashwagandha supplementation may moderate the body's adaptation in response to resistance training. While this hypothesis is rooted in traditional Ayurvedic medicine and previous studies, well designed clinical trials are clearly needed to test and characterize the effects. The present double-blind, randomized, placebo-controlled study in healthy adults will, it is hoped, help toward expanding scientific understanding in this domain.

\section{Methods}

The study design, recruitment and methods were approved by all the authors' institutes' review boards on human subjects' studies and followed the guidelines of the Declaration of Helsinki and Tokyo for humans.

\section{Subjects selection, incentives and participation}

Healthy male subjects (18-50 years old) were recruited by use of fliers circulated in the vicinity of the gymnasium which served as the site of the training program. Subject enrollment, allocation, attrition, and analysis is summarized in Fig. 1. The purpose and protocol for the study was described to the subjects. The subjects had to 


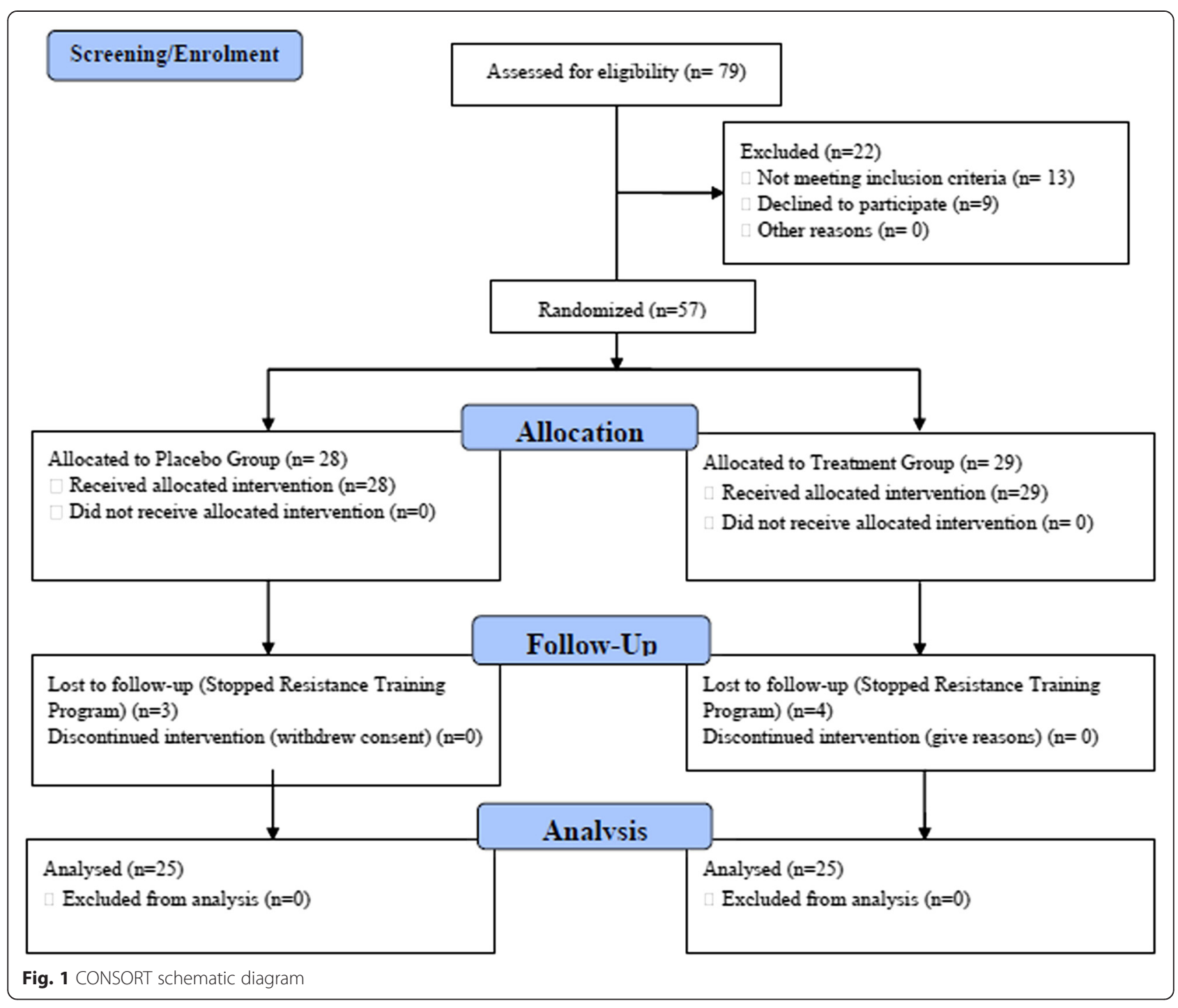

sign a written consent for the study, agree to refrain from alcohol and tobacco during the study, and had to receive permission of their physician to participate. Subjects were excluded from the study if they met any of the following criteria: 1) taking any medication or steroids to enhance physical performance, 2) weight loss of $>5 \mathrm{~kg}$ in the previous 3 months, 3) any history of drug abuse, smoking $10+$ cigarettes day or consuming more than 14 grams of alcohol daily, 4) hypersensitivity to ashwagandha, 5) history of any orthopedic injury or surgery within the previous 6 months, 6) participation in other clinical studies during the previous 3 months, 7) any other conditions which the investigators judged problematic for participation in the study. Subjects were requested to refrain from using anti-inflammatory agents during the study and to report any ill effects from consuming the ashwagandha/ placebo.
Because participation in the study would require a significant amount of time allocation from the subjects, the recruiters offered as compensation one year of paid membership to the gym and three months of professional trainer support. As Fig. 1 indicates, 57 subjects were initially recruited for the study and 50 completed the study, including 25 in the ashwagandha group and 25 in the placebo group. For the ashwagandha treatment group and for the placebo group, the mean age \pm standard deviation were $28 \pm 8$ years and $29 \pm 9$ years, respectively.

\section{Study design}

This study was a prospective, double-blind, placebo-controlled parallel group study to measure the possible effects of ashwagandha extract on muscle strength/size, muscle recovery, testosterone level and body fat percentage in 
young males undergoing weight training. Adverse events were assessed by patient/ researcher reporting and the PGATT (Physicians Global Assessment of Tolerability to Therapy) form.

\section{The resistance training program}

The resistance training program consisted of sets of exercises over major muscle groups in both the upper body and the lower body. Directions for the resistance training were obtained from publications of the National Strength and Conditioning Association (NSCA) [31-33]. Each subject in both groups was asked to come to a training session every other day, with one rest day per week, for three days per week. Every session began with a warm up consisting of five minutes of low-intensity aerobic exercise.

The subjects were instructed to perform, for each set, as many repetitions as they could until failure. The subjects were asked to go through the full range of motion and were demonstrated the proper technique for safe and effective weight lifting.

\section{Exercise selection}

The specific exercises and the number of sets in each session were as follows. For the first week, the subjects were asked to perform the barbell squat ( 2 sets), the leg extension ( 1 set), the seated leg curl ( 2 sets), the machine chest press $(1 \mathrm{set})$, the barbell chest press (2 sets), the seated machine row (1 set), the one-arm dumbbell row ( 2 sets), the machine biceps curl (1 set), the dumbbell biceps curl ( 2 sets), the cable triceps press-down (2 sets), the dumbbell shoulder press ( 2 sets), and the straight-arm pull-down ( 2 sets). For the second week, the subjects were asked to perform the leg extension (1 set), the barbell squat ( 2 sets), the barbell chest press ( 3 sets), the seated leg curl ( 2 sets), the seated cable row (3 sets), dumbbell biceps curl ( 3 sets), the cable triceps press-down ( 3 sets), the dumbbell shoulder press ( 3 sets), and the straight-arm pull-down or lat pull-down (3 sets). After this two-week acclimatization phase, for the rest of the study, the subjects were asked to perform the barbell squat ( 3 sets), the leg extension ( 3 sets), the leg curl ( 2 sets), one chest exercise (flat, incline or decline press or fly, cable crossover, 3 sets), one back exercise (rows, pull up, pull down or seated cable row, 3 sets), another chest exercise (3 sets), another back exercise (3 sets), one biceps exercise or one triceps exercise (curls or extensions, 3 sets), and one shoulder exercise (raises or presses, 3 sets).

\section{How the target number of repetitions was chosen}

The number of repetitions for the initial two weeks was set to be 15 , which is a moderately high number (implying correspondingly lower weights), chosen to allow a subject's body and neural system to get accustomed to strength training $[31,33]$. The subsequent 6 weeks had a varying number of repetitions, akin to a rudimentary nonlinear periodization programs, because these have been shown to induce greater adaptation and more gains in muscle strength and size [33]. The number of repetitions specified for each of the days in the training program is given in Table 1.

\section{How the load was chosen}

The load was chosen to be such that the subject would reach the failure-point upon performing approximately the target number of repetitions (chosen as described in the preceding paragraph) when lifting that load. The corresponding load for an exercise was estimated on the basis of the 1RM prediction equations of Epley, Wathan and others [34].

\section{Treatments and dosing}

The researchers engaged a local laboratory to fill cellulose-based vegetarian capsules with either $300 \mathrm{mg}$ of starch or $300 \mathrm{mg}$ of a high-concentration ashwagandha root extract, KSM-66, manufactured by Ixoreal BioMed, Los Angeles, California, USA. This extract was produced using a water-based process using no alcohol or solvents and is standardized to a $5 \%$ concentration of withanolides as measured by HPLC. The two capsules were identical in appearance, weight, and texture. Both the control and ashwagandha groups received a bottle of 60 pills at start of study and at 4 weeks. The pill count at 4 weeks allowed for a compliance check. The subjects were instructed to store the capsules between 18 and $32{ }^{\circ} \mathrm{C}$ and to take the capsules twice a day, once shortly after awakening and again shortly before bed, for the 8 weeks of the study.

\section{Primary efficacy endpoint}

The primary efficacy endpoint was muscle strength. Muscle strength is often measured by $1 \mathrm{RM}$, the "onerepetition maximum", which is specific to a certain person and a certain exercise movement and refers to the maximal load that a subject can lift for one movement cycle of the exercise [31,35]. Measurements were made at the first day of training and again 2 days after the 8 week training ended. The equipment used machine models DPL0802 (bench press) and DSL0605 (leg extension),

Table 1 The targeted number of repetitions over the course of the resistance training program

\begin{tabular}{lllll}
\hline & Weeks 1-2 & Weeks 3-4 & Weeks 5-6 & Weeks 7-8 \\
\hline Day 1 & 15 & 13 & 5 & 9 \\
Day 2 & 15 & 9 & 13 & 5 \\
Day 3 & 15 & 13 & 5 & 9 \\
Day 4 & 15 & 9 & 13 & 5 \\
\hline
\end{tabular}


manufactured by Precor (Woodinville, Washington, USA The 1-RM measurement was done using a variant of the widely used Baechle-Earle-Wathen protocol, employing the multiple RM method [36-40]. To reduce measurement error in strength assessment, we were careful to ensure consistency in the range of motion and that each movement on the chest press and the leg extension was complete and in accordance with the guidelines of the NSCA.

\section{Secondary efficacy endpoints}

The secondary efficacy endpoints related to serum testosterone level, muscle recovery and anthropometric factors capturing muscle size and body fat percentage.

\section{Anthropometry}

Muscle size: Muscle size was measured at 3 sites: the arm (flexed mid upper arm), chest (sternum at mid-tidal volume) and upper thigh (just inferior to gluteal fold). Measurements were done on the first day of the training period and 2 days after the last day of training. For the chest, we measured the girth, taken at the level of the middle of the sternum, with the tape passing under the arms and at the end of a normal expiration. For the thigh and arm, we assessed the maximal cross-sectional area (CSA) using the method of Moritani-DeVries, which is based on girth and skin-fold measurements [41-43]. The literature shows that the muscle CSA measures obtained by the Moritani-DeVries method are highly correlated with measures obtained by computer tomography or muscle biopsy, the gold standards for muscle CSA measurement [41-43]. Because of this high correlation, the across-time (Day 0 versus Day 56) or across-group (treatment versus placebo) comparisons on the basis of the Moritani-DeVries method are strongly indicative of the directionality and strength of the corresponding comparisons on the basis of the computer tomography. We chose to use the Moritani-DeVries method because it is less time-consuming and invasive than computer tomography or biopsy, and therefore less likely to discourage study participation.

\section{Body fat percentage}

Body fat percentage was calculated with a bioelectrical impedance method using machine with electrodes placed at the hand, wrist, foot, and ankle [44, 45]. Because bioelectrical impedance analysis based measurement of fat composition is known to be affected by extraneous factors like hydration level and temperature, we tried to maintain consistency in these factors by instructing the subjects to: 1) abstain from eating or drinking for $4 \mathrm{~h}$ before measurements, 2) urinate $30 \mathrm{~min}$ prior, 3) not engaged in exercise for $12 \mathrm{~h}$ prior and 4) not consume alcohol of caffeinated products [35, 45].
Body composition was measured two days after the first day of resistance training and again two days after the last day of resistance training.

\section{Testosterone}

Total blood testosterone serum levels were measured twice: once 2 days after the study commenced and again 2 days after the study ended. The blood draw was timed to be between two hours and three hours of each subject's regular waking time, and prior to any substantial physical activity, in order to minimize the effects of the natural diurnal variation in testosterone level. The $20 \mathrm{ml}$ blood draws were from an antecubital vein, punctured with a 20-gauge disposable needle connected to a Vacutainer tube. The blood serum samples were analyzed by an ELISA (enzyme-linked immunosorbent assay).

\section{Muscle recovery}

Resistance training frequently damages skeletal muscle tissue. Such damage can result in decreased muscle force production and performance in subsequent training sessions, thereby possibly reducing the extent of adaptation and gains from resistance training [46]. Muscle recovery refers to the reduction in exercise-induced muscle damage over time. The level of creatine kinase, a protein, in the blood is a commonly used measure of muscle damage because this protein is specific to muscle tissue [47]. When muscles are overexerted, the muscle filaments are damaged and become necrotic, thereby causing soluble proteins like creatine kinase to migrate from muscle tissue into the blood stream [48]. The body on its own repairs such damage over 1 to 10 days and serum creatine kinase returns to baseline levels [48]. A bout of exercise tends to produce less damage in muscle tissue when repeated in subsequent training sessions after the body gets accustomed to the exercise. This is because of adaptation and strengthening of the muscle tissue. Serum creatine kinase was measured at $24 \mathrm{~h}$ and at $48 \mathrm{~h}$ after the end of the first exercise session, and also at $24 \mathrm{~h}$ and at $48 \mathrm{~h}$ after the end of the last exercise session approximately 8 weeks later, from $20 \mathrm{ml}$ blood draws using a 20-gauge disposable needle and a Vacutainer setup. The creatine kinase level was determined in a commercial laboratory using enzymatic analysis tracking nicotinamide adenine diphesphopyridine (NADPH). The increase in creatine kinase from the $24-\mathrm{h}$ point to the 48 -h point can be taken as a biomarker of recovery in that a smaller increase corresponds to faster stabilization of creatine kinase level and hence faster recovery of muscle tissue from exercise-induced damage.

\section{Tolerability}

The subjects were asked to report any adverse events experienced at any point in the study. We used the 
Physicians Global Assessment of Tolerability to Therapy (PGATT) form [49-51]. Subjects used a five-point scale to assess tolerability from "worst tolerability" (which corresponds to patients' not being able to tolerate the drug at all) to "excellent tolerability" (which corresponds to no adverse effects and the patient being able to tolerate the drug excellently).

\section{Statistical analysis}

Assessment of statistical significance of continuous treatment effects was done using ANOVA with group identity (treatment versus placebo) as a factor. We used the Mann-Whitney test if the data were found to be not normally distributed. Frequencies of the tolerability scale values were compared using the chi-square test for contingency tables. The accepted level of significance was $\alpha=0.05$.

\section{Results}

Tables 2, 3, 4, 5 and 6 compare the treatment group and the placebo group at baseline, at the start of the study and at the end of the 8 week study for the following 5 factors: testosterone level $(\mathrm{ng} / \mathrm{dL})$, muscle strength on the bench press 1-RM $(\mathrm{Kg})$, muscle strength on the leg extension 1-RM $(\mathrm{Kg})$, muscle size at thighs $\left(\mathrm{cm}^{2}\right)$, muscle size at arms $\left(\mathrm{cm}^{2}\right)$, muscle size at chest $(\mathrm{cm})$, body fat percentage, muscle recovery in terms of creatine kinase levels change (U/L),

\section{Primary efficacy measure: muscle strength}

There was a significant increase in muscle strength and muscle size in both the ashwagandha group and the placebo group, for both the upper and lower body. This is unsurprising because both group engaged in resistance training. The focal question is whether the adaptation is greater under ashwagandha supplementation. Table 2 and Fig. 2 show that the increases in muscle strength were statistically significantly greater in the ashwagandha group than in the placebo group, for the upper body (Placebo: $26.42 \mathrm{~kg}, 95 \% \mathrm{CI}, 19.52,33.32$ vs. Ashwagandha: $46.05 \mathrm{~kg}, 95 \%$ CI 36.56, 55.54; $p=0.001$ ) and the lower body (Placebo: $9.77 \mathrm{~kg}, 95 \% \mathrm{CI}, 7.18,12.35$ vs. Ashwagandha: $14.50 \mathrm{~kg}, 95 \%$ 10.76, 18.23; $p=0.04$ ).

\section{Secondary efficacy measures Anthropometry}

Muscle Size: For muscle size (Table 3; Fig. 2), the increases are significantly greater in the ashwagandha group than the placebo group in the arm (Placebo: 5.30 $\mathrm{cm}^{2}, 95 \% \mathrm{CI}, 3.34,7.25$ vs. Ashwagandha: $8.89 \mathrm{~cm}^{2}$, $95 \%$ 6.95,10.84; $p=0.01$ ) and chest (Placebo: $1.43 \mathrm{~cm}$, 95 \% CI, 0.83, 2.02 vs. Ashwagandha: $3.37 \mathrm{~cm}, 95$ \% CI, $2.59,4.15 ; p<0.001$ ) but not in the thighs (Placebo: 6.22 $\mathrm{cm}^{2}, 95 \% \mathrm{CI}, 2.61,9.84$ vs. Ashwagandha: $8.71 \mathrm{~cm}^{2}$, $95 \%$ CI, 4.56, 12.87; $p=0.36$ ).

Body composition: Table 4 shows that body fat percentages declined in both groups over the 8 week study, with the fat percentage decrease being significantly greater among subjects in the ashwagandha group as compared to the placebo group (Placebo: $1.52 \%, 95 \% \mathrm{CI}, 0.46,2.59$, vs. Ashwagandha: $3.47 \%$, $95 \%$ CI, 1.99, 4.95; $p=0.03$ ).

\section{Serum testosterone}

Over the eight weeks, there was a significant increase in testosterone level in the ashwagandha treatment group relative to the placebo group (Table 5; Fig. 3). The increase in testosterone level was significantly greater with ashwagandha supplementation than with the placebo (Placebo: 18.00 ng/dL, 95 \% CI, -15.83, 51.82 vs. Ashwagandha: $96.19 \mathrm{ng} / \mathrm{dL}, 95 \% \mathrm{CI}, 54.86,137.53 ; p=0.004$ ). While the mean post-intervention level was notably higher in the ashwagandha group than in the placebo group (726 versus 693), the numbers are not detectable as statistically significantly different, very likely because the across-subject variance is high.

\section{Muscle recovery}

Recall that the level of recovery from exercise-induced muscle damage is assessed through the increase in level of serum creatine kinase from Hour 24 to Hour 48 after the end of the resistance training session. A smaller increase in this muscle protein in the blood stream

Table 2 Muscle strength

\begin{tabular}{|c|c|c|c|c|}
\hline & & Treatment group & Placebo group & Between group comparison \\
\hline & & Mean (SD) & Mean (SD) & ( $p$-values) \\
\hline & Sample size $(n)$ & $n=25$ & $n=25$ & \\
\hline \multirow[t]{3}{*}{ Bench Press 1RM (Kg) } & Pre intervention & $33.21(8.50)$ & $31.35(7.97)$ & 0.44 \\
\hline & Post intervention & $79.26(25.90)$ & $57.77(16.48)$ & 0.001 \\
\hline & Change & 46.05 (23.00); 95 \% Cl: 36.56, 55.54** & 26.42 (16.72); 95 \% Cl: $19.52,33.32^{* *}$ & 0.001 \\
\hline \multirow[t]{3}{*}{ Leg Extension 1RM (Kg) } & Pre intervention & $27.89(4.24)$ & $25.22(7.03)$ & 0.11 \\
\hline & Post intervention & $42.38(10.80)^{* *}$ & $34.98(10.54)^{* *}$ & 0.02 \\
\hline & Change & 14.50 (9.04); 95 \% Cl: 10.76, 18.23** & 9.77 (6.27); 95 \% Cl: $7.18,12.35^{* *}$ & 0.04 \\
\hline
\end{tabular}

${ }^{* *}=p<0.001$ within group comparison 
Table 3 Muscle size

\begin{tabular}{lllll}
\hline & & Treatment group mean (SD) & Placebo group mean (SD) & $\begin{array}{l}\text { Between group comparison } \\
(p \text {-values })\end{array}$ \\
\hline \multirow{3}{*}{ Thigh $\left(\mathrm{cm}^{2}\right)$} & Sample size $(\mathrm{n})$ & $n=25$ & $n=25$ & 0.58 \\
& Pre intervention & $107.84(24.61)$ & $111.18(17.15)$ & 0.9 \\
& Post intervention & $116.56(26.04)$ & $117.40(19.96)$ & 0.36 \\
& Change & $8.71(10.06) ; 95 \% \mathrm{Cl}: 4.56,12.87^{* *}$ & $6.22(8.76) ; 95 \% \mathrm{Cl}: 2.61,9.84^{*}$ & 0.75 \\
Arm $\left(\mathrm{cm}^{2}\right)$ & Pre intervention & $51.96(10.88)$ & $53.13(14.84)$ & 0.59 \\
& Post intervention & $60.85(13.23)$ & $58.43(17.66)$ & 0.01 \\
& Change & $8.89(4.71) ; 95 \% \mathrm{Cl}: 6.95,10.84^{* *}$ & $5.30(4.74) ; 95 \% \mathrm{Cl}: 3.34,7.25^{* *}$ & 0.93 \\
Chest $(\mathrm{cm})$ & Pre intervention & $101.40(11.22)$ & $101.16(8.93)$ & $102.58(8.76)$ \\
& Post intervention & $104.77(11.09)$ & $1.43(1.45) ; 95 \% \mathrm{Cl}: 0.83,2.02^{* *}$ & 0.0002 \\
\hline
\end{tabular}

${ }^{*}=p<0.01 ;{ }^{* *}=p<0.001$ within group comparison

corresponds to faster muscle tissue repair, which in turn corresponds to greater recovery. Table 6 and Fig. 3 show how this metric varied across groups and over time. It is important to keep in mind that smaller numbers are to be interpreted as better recovery. What is striking is that recovery was dramatically better after 8 weeks of resistance training, in both the ashwagandha group and the placebo group, likely because of muscle tissue getting accustomed to the training regimen and developing greater integrity to resist any damage. Comparing the ashwagandha group and the placebo group, the results showed that recovery is substantially higher in the ashwagandha group than in the placebo group (Placebo: 1307.48 U/L, $95 \%$ CI, $-1202.82,1412.14$ vs. Ashwagandha: 1462.68 U/ L, 95 \% CI, 1366.27, 1559.09; $p=0.03$ ).

\section{Tolerability}

No serious side effects were reported by subjects in either group. All subjects rated tolerability as either "good" or "excellent" on the PGATT form. There was no statistically significant difference in PGATT scores between the 2 groups.

Table 4 Body fat percentage

\begin{tabular}{|c|c|c|c|}
\hline & Treatment group & Placebo & $\begin{array}{l}\text { Between group } \\
\text { comparison }\end{array}$ \\
\hline & Mean (SD) & Group mean (SD) & ( $p$-values) \\
\hline $\begin{array}{l}\text { Sample } \\
\text { size (n) }\end{array}$ & $n=25$ & $n=25$ & \\
\hline Pre intervention & $21.60(3.91)$ & $22.01(3.37)$ & 0.7 \\
\hline Post intervention & $18.13(3.13)^{* *}$ & $20.48(1.85)^{*}$ & 0.003 \\
\hline Change & $\begin{array}{l}-3.47(3.58) ; 95 \% \\
\text { Cl: }-4.95,-1.99^{* *}\end{array}$ & $\begin{array}{l}-1.52(2.58) ; 95 \% \\
\text { Cl: }-2.59,-0.46^{*}\end{array}$ & 0.03 \\
\hline
\end{tabular}

${ }^{*}=p<0.01 ;{ }^{* *}=p<0.001$ within group comparison

\section{Discussion}

This is the first research paper that we know of that studies ashwagandha as an adjuvant to resistance training programs. Because subjects in both the ashwagandha group and the placebo group engaged in resistance training, we would expect to see a substantial degree of improvement in muscle-related parameters in both groups, and indeed we did. These findings are consistent with numerous studies which have measured adaptation to strength training programs in the absence of any supplementation [33]. The focal question that we sought to examine in this research is related to whether ashwagandha supplementation would magnify these adaptations. The adaptations were found to be statistically significantly greater, at a $p$-value threshold of 0.05 , with ashwagandha supplementation than under placebo for all parameters (muscle strength, muscle size and body fat percentage, testosterone, and muscle recovery) except for thigh muscle size, though some effects were marginal. Increased recovery from muscle damage has the practical implication that it allows one to resume resistance training more quickly, thereby increase the volume of training per unit time and thereby potentially achieve

Table 5 Serum testosterone level (ng/dL)

\begin{tabular}{|c|c|c|c|}
\hline & $\begin{array}{l}\text { Treatment } \\
\text { group }\end{array}$ & $\begin{array}{l}\text { Placebo } \\
\text { group }\end{array}$ & $\begin{array}{l}\text { Between } \\
\text { group } \\
\text { comparison }\end{array}$ \\
\hline & Mean (SD) & Mean (SD) & (p-values) \\
\hline $\begin{array}{l}\text { Sample } \\
\text { size }(n)\end{array}$ & $n=25$ & $n=25$ & \\
\hline Pre intervention & $630.45(231.88)$ & $675.12(157.02)$ & 0.43 \\
\hline Post intervention & $726.64(171.55)^{* *}$ & $693.12(115.04)$ & 0.42 \\
\hline Change & $\begin{array}{l}96.19(100.14) ; 95 \% \\
C l: 54.86,137.53^{* *}\end{array}$ & $\begin{array}{l}18.00(81.94) ; 95 \% \\
C l:-15.83,51.82\end{array}$ & 0.004 \\
\hline
\end{tabular}

** $=p<0.001$ within group comparison 
Table 6 Muscle recovery: increase of serum creatine kinase from hour 24 to hour 48 after end of exercising (U/L)

\begin{tabular}{llll}
\hline & Treatment group & Placebo group & Metween group comparison \\
& Mean (SD) & Mean (SD) & $(p$-values $)$ \\
\hline Sample size $(\mathrm{n})$ & $n=25$ & $n=25$ & 0.31 \\
Pre intervention & $1478.88(239.60)$ & $1406.52(264.45)$ & $<0.0001$ \\
Post intervention & $16.20(9.47)^{* *}$ & $99.04(16.77)^{* *}$ & 0.03 \\
Change & $-1462.68(233.57) ; 95 \%$ Cl: $-1559.09,-1366.27^{* *}$ & $-1307.48(253.54) ; 95 \%$ Cl: $-1412.14,-1202.82^{* *}$ & \\
\hline
\end{tabular}

$*^{* *}=p<0.001$ within group comparison

greater gains per unit time. Our basic results are consistent with the findings of previous human studies with ashwagandha $[8,9,28,29,52]$ in demonstrating gains in muscle strength, body composition and testosterone, though these other studies do not follow these parameters all in a single clinical trial or in conjunction with a resistance training program. The ashwagandha extract was tolerated very well at the study dose with no side effects reported. This good safety profile of ashwagandha is consistent with reports from previous studies [8, 9, 28, 29, 52].

There are also studies considering the use of other adaptogenic herbs like Rhodiola rosea, Eleutherococcus senticosus, Schisandra chinensis and ginseng toward physical performance. One study [53] gave evidence that Rhodiola rosea supplementation can improve endurance and reduce time to exhaustion. A review of Russian research [15] identifies human studies that show improved physical and mental performance from Schisandra supplementation. It is suggested that [15] Schisandra supplementation can help elite athletes adapt to high physical intensities. More study of the use of adaptogen herbs in the aid of muscle strength and recovery is needed.

There are several mechanisms of action that could have contributed to the positive effects of ashwagandha supplementation on resistance training and performance improvements in this study. These can be viewed from two perspectives: muscle development and muscle recovery.

\section{Muscle development}

The ability to lift weights is a function of (a) muscle size, (b) energy production and (c) the nervous system's

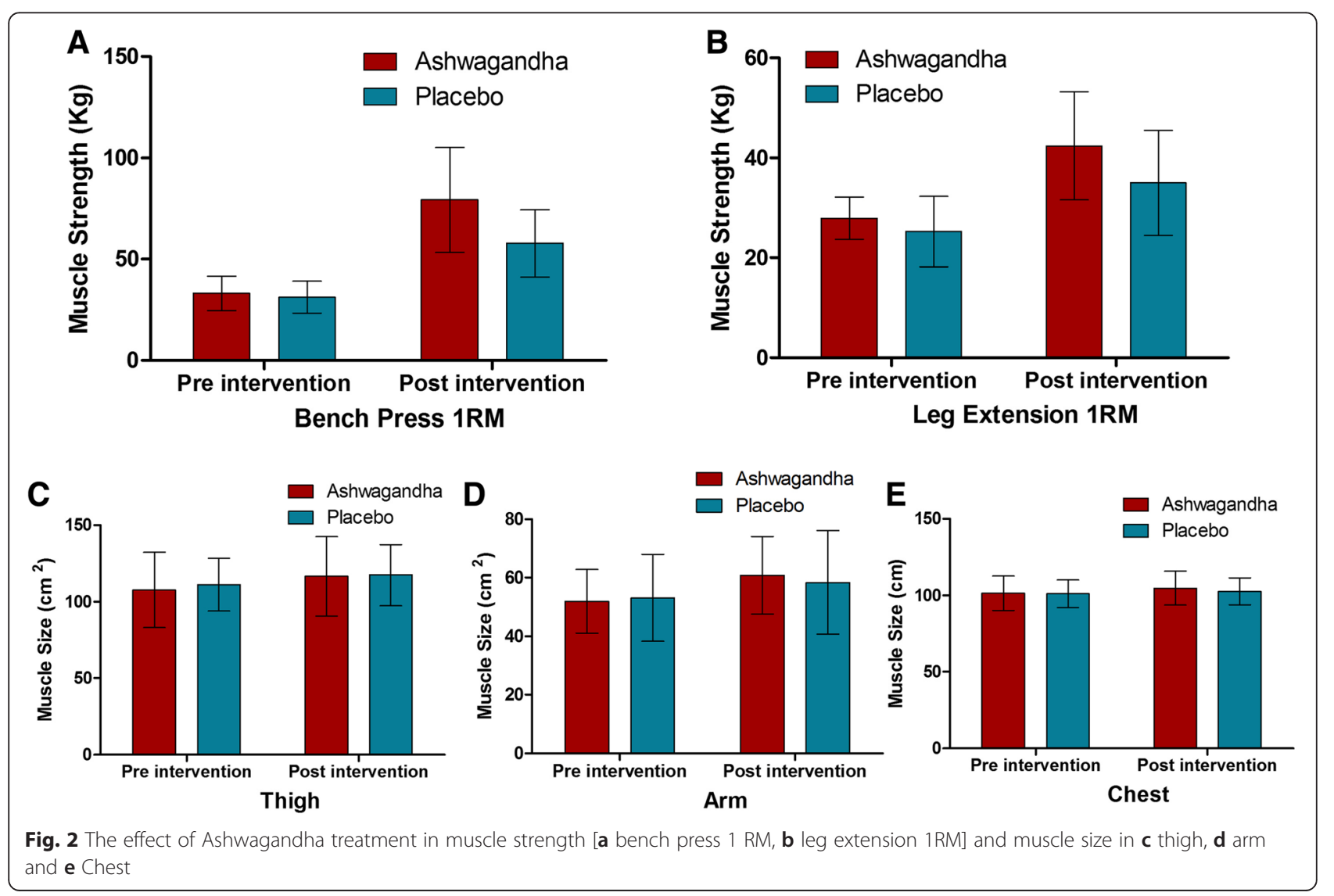



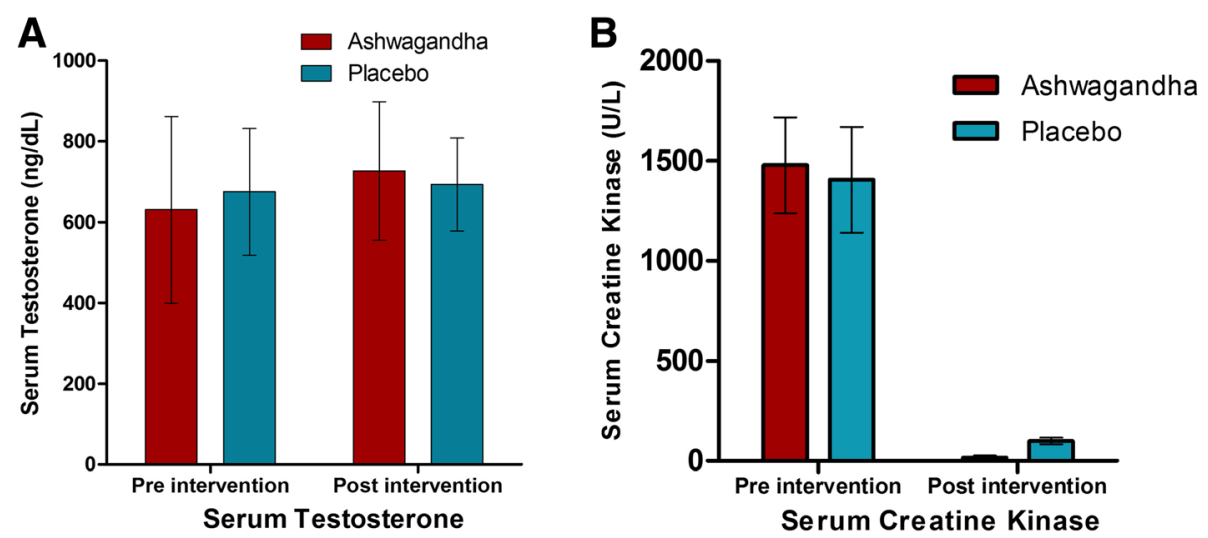

Fig. 3 The effect of Ashwagandha treatment in a serum testosterone and $\mathbf{b}$ serum creatine kinase level

ability to recruit muscles and coordinate them to generate the required force. Muscle size is a function of muscle growth, which is affected by two of ashwagandha's effects: (i) increase in testosterone, which leads to muscle growth and (ii) decrease in the levels of cortisol, which as a catabolic agent detracts from muscle mass. In terms of energy production, ashwagandha (i) can have beneficial effects on mitochondrial energy levels and functioning and reduce the activity of the $\mathrm{Mg} 2$ + -dependent ATPase enzyme responsible for the breakdown of ATP [54], and (ii) can increase creatine levels that can in turn lead to ATP generation [8]. Finally, the effects of ashwagandha on the nervous system as antianxiety agent and in promoting focus and concentration [28] may translate to better coordination and recruitment of muscles. The reason for the lack of an effect on thigh size is not clear. Longer term studies are needed to shed light on this, as are studies looking at markers in the local environment of these muscles to rule out any biochemical anomalies as contributing factors.

\section{Muscle recovery}

In the present study, more rapid recovery from muscle damage under supplementation with ashwagandha was demonstrated by monitoring creatine kinase. The faster recovery could be due to a number of mechanisms, or more likely their synergistic effects, as mediated by the various extract components, such as antioxidant effects to combat free radical damage both at the muscle and central nervous system levels, anti-inflammatory and analgesic effects and reduction in lactic acid and blood urea nitrogen [4-6]. In that vein, muscle soreness is a common occurrence following exercise for those less accustomed to physical activity. Delayed onset muscle soreness (DOMS) presents between 24 and $48 \mathrm{~h}$ after exercise as tenderness to palpation, and/or movement accompanied by decreases in flexibility and maximal voluntary force production. This soreness can inhibit full and proper exercise. Thus, a reduction in DOMS as a consequence of ashwagandha's effect on reduced muscle injury would counteract this negative consequence.

\section{Conclusion}

This study confirms previous data regarding the adaptogenic properties of ashwagandha and suggests it might be a useful adjunct to strength training. This study has the following limitations which should lead us to interpret the findings with some caution: the subjects are untrained and moderately young, the sample size of 50 is not large and the study period is of duration only 8 weeks. Research studying the possible beneficial effects of ashwagandha needs to be conducted for longer periods of time and for different populations including females and older adults of both genders.

\section{Competing interests}

The authors declare that there is no conflict of interests regarding the publication of this paper.

\section{Authors' contributions}

SW and DL developed the clinical trial design and the resisitance training protocol. They carried out the data collection in collaboration with $\mathrm{KJ}$ and SS. SW, DL, KJ and SS oversaw the data treatment and data analysis. SB contributed to the writing, the presentation, the bibliography and managed correspondence. All authors were involved in the writing and drafting, and all read and approved the final manuscript.

\section{Acknowledgments}

The authors thank Shri Kartikeya Pharma and Ixoreal BioMed of Los Angeles, California, USA for supplying the KSM-66 high-concentration root extract used in the study treatment.

\section{Author details}

${ }^{1}$ Sports Medicine, SrimatiKashibaiNavale Medical College, Pune, India. 2Department of Pharmacology, BVDU Dental College \& Hospital, Navi Mumbai, India. ${ }^{3}$ Department of Pharmacology, BharatiVidyapeeth Medical College \& Hospital, Sangli, India. ${ }^{4}$ Department of Clinical Pharmacology, Grant Government Medical College, SirJamshedjeeJeejeebhoy Group of Hospitals, Mumbai, India. ${ }^{5}$ Department of Pharmaceutical Technology, NSHM Knowledge Campus, 124 B.L. Saha Road, Kolkata 700053, India.

Received: 13 July 2015 Accepted: 16 November 2015

Published online: 25 November 2015 


\section{References}

1. Williamson EM et al. Major Herbs of Ayurveda. London: Churchill Livingstone; 2002.

2. Kapoor LD. CRC Handbook of ayurvedic medicinal plants. Carbohydr: Boca Raton, Florida; 1990.

3. Upton R. Ashwagandha Root: Withania Somnifera: Analytical, Quality Control, and Therapeutic Monograph. Santa Cruz, California: American Herbal Pharmacopoeia; 2000.

4. Mishra LC, Singh BB, Dagenais S. Scientific basis for the therapeutic use of Withania somnifera (ashwagandha): a review. Alternative Medicine Review. 2000;5:334-46.

5. Vyas VK, Bhandari P, Patidar R. A Comprehensive Review on Withania somnifera Dunal. J Nat Remedies. 2011;11:1-13.

6. Singh G, Sharma PK, Dudhe R, Singh S. Biological activities of Withania somnifera. Ann Biol Res. 2010;1:56-63.

7. Braun L, Cohen M. Herbs and Natural Supplements, Volume 1: An EvidenceBased Guide. Elsevier Health Sciences; 2015.

8. Raut A, Tadvi F, Kene K, Pandey S, Vaidya A, Rege N, Solanki P, Shirolkar S, Vaidya R: Exploratory study to evaluate tolerability, safety, and activity of Ashwagandha (Withania somnifera) in healthy volunteers. J Ayurveda Integrative Med 2012:111.

9. Sandhu JS, Shah B, Shenoy S, Chauhan S, Lavekar GS, Padhi MM. Effects of Withania somnifera (Ashwagandha) and Terminalia arjuna (Arjuna) on physical performance and cardiorespiratory endurance in healthy young adults. Int J Ayurveda Res. 2010;1:144-9.

10. Shenoy S, Chaskar U, Sandhu JS, Paadhi MM. Effects of eight-week supplementation of Ashwagandha on cardiorespiratory endurance in elite Indian cyclists. J Ayurveda Integr Med. 2012;3:209-14

11. Sastry JLN. Ayurvedokta Oushadha niruktamala. Edn 1st, Chaukhambha Orient Varanasi 2001, 36.

12. Abascal K, Yarnell E. Increasing vitality with adaptogens: multifaceted herbs for treating physical and mental stress. Altern Complement Ther. 2003;9:5460.

13. Bagchi D, Nair S, Sen CK. Nutrition and Enhanced Sports Performance: Muscle Building, Endurance, and Strength. Academic Press; 2013.

14. Molinos Domene Á. Effects of adaptogen supplementation on sport performance. A recent review of published studies. 2013.

15. Panossian A, Wikman G. Pharmacology of Schisandra chinensis Bail.: an overview of Russian research and uses in medicine. J Ethnopharmacol. 2008; 118:183-212.

16. Kraemer WJ, Ratamess NA. Fundamentals of resistance training: progression and exercise prescription. Med Sci Sports Exerc. 2004;674688.

17. American College of Sports Medicine. American College of Sports Medicine position stand. Progression models in resistance training for healthy adults. Med Sci Sports Exerc. 2009;41:687.

18. Sale DG. Neural adaptation to resistance training. Med Sci Sports Exerc. 1988;20(5 Suppl):S135-45.

19. Staron RS, Karapondo DL, Kraemer WJ, Fry AC, Gordon SE, El FJ, et al. Skeletal muscle adaptations during early phase of heavy-resistance training in men and women. J Appl Physiol. 1994;76:1247-55.

20. Singh N, Nath R, Lata A, Singh SP, Kohli RP, Bhargava KP: (Ashwagandha), a Rejuvenating Herbal Drug Which Enhances Survival During Stress (an Adaptogen). Pharmaceutical Biol 1982:29-35.

21. Kulkarni SK, Dhir A. Withania somnifera: An Indian ginseng. Progress in Neuro-Psychopharmacology and Biological Psychiatry 2008:1093-1105.

22. Davis L, Kuttan G. Effect of Withania somnifera on cyclophosphamideinduced urotoxicity. Cancer Lett. 2000;148:9-17.

23. Davis L, Kuttan G. Suppressive effect of cyclophosphamide-induced toxicity by Withania somnifera extract in mice. J Ethnopharmacol. 1998;62:209-14.

24. Sharma S, Dahanukar SA, Karandikar SM. Effects of long term administration of the roots of ashwagandha and shatavari in rats. 1985.

25. Archana R, Namasivayam A. Antistressor effect of Withania somnifera. J Ethnopharmacol. 1998;64:91-3.

26. Prakash J, Gupta SK, Dinda AK. Withania somnifera root extract prevents DMBA-induced squamous cell carcinoma of skin in Swiss albino mice. Nutr Cancer. 2002;42:91-7.

27. Singh A, Naidu PS, Gupta S, Kulkarni SK. Effect of natural and synthetic antioxidants in a mouse model of chronic fatigue syndrome. J Med Food. 2002;5:211-20.
28. Chandrasekhar K, Kapoor J, Anishetty S. A prospective, randomized doubleblind, placebo-controlled study of safety and efficacy of a highconcentration full-spectrum extract of ashwagandha root in reducing stress and anxiety in adults. Indian J Psychol Med. 2012;34:255-62.

29. Ambiye VR, Langade D, Dongre S, Aptikar P, Kulkarni M, Dongre A. Clinical evaluation of the spermatogenic activity of the root extract of Ashwagandha (Withania somnifera) in oligospermic males: a pilot study. Evidence-based Complement Altern Med. 2013;2013:571420.

30. Bhattacharya SK, Satyan KS, Ghosal S. Antioxidant activity of glycowithanolides from Withania somnifera. Indian J Exp Biol. 1997;35: 236-9.

31. Baechle TR, Earle RW. Essentials of Strength Training and Conditioning. 2008. Human kinetics.

32. Commission NC et al. Exercise Technique Manual for Resistance Training, Human Kinetics. 2008.

33. Fleck SJ, Kraemer W. Designing Resistance Training Programs, 4E, Human Kinetics. 2014.

34. LeSuer DA, McCormick JH, Mayhew JL, Wasserstein RL, Arnold MD. The accuracy of prediction equations for estimating 1-RM performance in the bench press, squat, and deadlift. J Strength Cond Res. 1997;11:211-3.

35. McArdle WD, Katch Fl, Katch VL. Exercise Physiology: Nutrition, Energy, and Human Performance. Lippincott Williams \& Wilkins; 2010.

36. Mayhew JL, Ball TE, Bowen JC. Prediction of bench press lifting ability from submaximal repetitions before and after training. Res Sport Med An Int J. 1992;3:195-201.

37. Shimano T, Kraemer WJ, Spiering BA, Volek JS, Hatfield DL, Silvestre R, et al Relationship between the number of repetitions and selected percentages of one repetition maximum in free weight exercises in trained and untrained men. J Strength Cond Res. 2006;20:819-23.

38. Reynolds JM, Gordon TJ, Robergs RA. Prediction of one repetition maximum strength from multiple repetition maximum testing and anthropometry. J Strength Cond Res. 2006;20:584-92.

39. Brzycki M. Strength testing-predicting a one-rep max from reps-to-fatigue. J Phys Educ Recreat Danc. 1993;64:88-90.

40. Dohoney P, Chromiak JA, Lemire D, Abadie BR, Kovacs C. Prediction of one repetition maximum (1-RM) strength from a 4-6 RM and a 7-10 RM submaximal strength test in healthy young adult males. J Exerc Physiol. 2002:5:54-9.

41. Moritani T et al. Neural factors versus hypertrophy in the time course of muscle strength gain. Am J Phys Med Rehabil. 1979;58:115-30.

42. DeFreitas JM, Beck TW, Stock MS, Dillon MA, Sherk VD, Stout JR, et al. A comparison of techniques for estimating training-induced changes in muscle cross-sectional area. J Strength Cond Res. 2010;24:2383-9.

43. Cholewa JM, Wyszczelska-Rokiel M, Glowacki R, Jakubowski H, Matthews T, Wood R, et al. Effects of betaine on body composition, performance, and homocysteine thiolactone. J Int Soc Sport Nutr. 2013;10:39.

44. Kraemer WJ, Fleck SJ, Deschenes MR. Exercise Physiology: Integrating Theory and Application. Lippincott Williams \& Wilkins; 2011

45. Nieman DC. Exercise Testing and Prescription: A Health Related Approach, McGraw-Hill Companies. 2003.

46. S. Thiebaud R. Exercise-induced muscle damage: is it detrimental or beneficial? J Quant Spectrosc Radiat Transf. 2012;1:36-44.

47. Clarkson PM, Hubal MJ. Are women less susceptible to exercise-induced muscle damage? Curr Opin Clin Nutr Metab Care. 2001;4:527-31.

48. Nosaka K. Exercise-induced muscle damage and delayed onset muscle soreness (DOMS). 2011

49. Jagade MV, Langade DG, Pophale RR, Prabhu A. Oxymetazoline plus dexpanthenol in nasal congestion. Indian J Otolaryngol Head Neck Surg 2008;60:393-7.

50. Kumar S, Rani S, Siwach R, Verma P. To compare the efficacy and safety of fixed dose combination of thiocolchicoside and aceclofenac versus chlorzoxazone, aceclofenac and paracetamol in patients with acute lower backache associated with muscle spasm. Int J Appl Basic Med Res. 2014:4:101.

51. Prabhoo R, Panghate A, Dewda RP, More B, Prabhoo T, Rana R. Efficacy and tolerability of a fixed dose combination of methylcobalamin and pregabalin in the management of painful neuropathy. N Am J Med Sci. 2012:4:605.

52. Mahdi AA, Shukla KK, Ahmad MK, Rajender S, Shankhwar SN, Singh V, et al Withania somnifera improves semen quality in stress-related male fertility. Evidence-based Complement Altern Med. 2011;2011:576962. 
53. De Bock K, Eijnde BO, Ramaekers M, Hespel P. Acute Rhodiola rosea intake can improve endurance exercise performance. Int J Sport Nutr Exerc Metab. 2004;14:298-307.

54. Begum VH, Sadique J. Effect of Withania somnifera on glycosaminoglycan synthesis in carrageenin-induced air pouch granuloma. Biochem Med Metab Biol. 1987;38:272-7.

Submit your next manuscript to BioMed Central and we will help you at every step:

- We accept pre-submission inquiries

- Our selector tool helps you to find the most relevant journal

- We provide round the clock customer support

- Convenient online submission

- Thorough peer review

- Inclusion in PubMed and all major indexing services

- Maximum visibility for your research 